\title{
CONCEPCIONES DE FUTUROS PROFESORES DE CIENCIAS SOBRE UN CONCEPTO «OLVIDADO» EN LA ENSEÑANZA DE LA ELECTRICIDAD: LA FUERZA ELECTROMOTRIZ
}

\author{
Guisasola, J. ${ }^{1}$; Montero, A. ${ }^{2}$ y Fernández, M. $^{3}$ \\ ${ }^{1}$ Departamento de Física Aplicada I. Euskal Herriko Unibertsitatea \\ ${ }^{2}$ Profesor de Física y Química del IES Sierra Almijara de Nerja \\ ${ }^{3}$ Departamento de Didáctica de las Ciencias Experimentales. Universidad de Granada
}

\begin{abstract}
Resumen. El trabajo que aquí se presenta recoge los resultados de una investigación en torno al aprendizaje del concepto de fuerza electromotriz a través de las siguientes etapas: a) conocer las principales dificultades que se produjeron en el desarrollo del concepto a lo largo de la historia; b) establecer las principales características de un aprendizaje significativo del concepto de fuerza electromotriz; c) averiguar en qué medida se presentan dificultades de aprendizaje de este concepto en estudiantes de último curso de carrera y recién licenciados en ciencias. Los resultados obtenidos parecen confirmar que los estudiantes, al acabar sus estudios de licenciatura no han logrado un claro significado de la magnitud fuerza electromotriz y, en consecuencia, presentan confusiones entre ésta y la diferencia de potencial en circuitos eléctricos y en fenómenos sencillos de inducción electromagnética. En la situación particular de un fenómeno sencillo de inducción electromagnética, casi la totalidad de los estudiantes presenta serias deficiencias en los significados de la fuerza electromotriz y diferencia de potencial.
\end{abstract}

Palabras clave. Fuerza electromotriz, diferencia de potencial, dificultades de aprendizaje, campo no conservativo, transferencia de energía.

Summary. This paper offers the result of our research into the learning of the concept of EMF at an introductory level according to the following stages: $a$ ) To know the main difficulties when defining the concept throughout history. $b$ ) To establish the main characteristics of the meaningful learning of the concept of EMF. c) To analyse to what extent students have a meaninful learning of EMF when they finish their degrees in science. The results seem to confirm that university students when finishing their science degrees have not got a clear idea of the notion of EMF. Therefore they often mistake it for potential difference. In the specific test of an easy experience of EMF induction, nearly all the students had serious problems in differentiating between EMF and potential difference.

Keywords. Electromotive force, potential difference, energy transfer, non-conservative field, learning difficulties.

\section{INTRODUCCIÓN}

El aprendizaje de los conceptos implicados en la correcta interpretación de distintos fenómenos eléctricos, diferencia de potencial, fuerza electromotriz, etc., así como la propia interpretación/explicación de los circuitos eléctricos es problemática. Desde hace una veintena de años, profesores y especialistas en didáctica de las ciencias experimentales investigan sobre el tema y celebran encuentros. La bibliografía especializada recoge los resultados de tales investigaciones: Driver y otros (1985), Hierrezuelo y Montero, (1989), Pfundt y Duit (1994).
La investigación didáctica pone de manifiesto que, en particular, los conceptos de potencial, campo y carga se aprenden mal desde los niveles de secundaria a los universitarios, afectando también a los profesores en ejercicio (Cohen et al., 1983; Duit y Jung, 1985; Furió y Guisasola 1997, 1998b; Solbes y Martín 1991; Montero 2002). A pesar de las propuestas didácticas que han demostrado su validez para superar en buena medida tales dificultades, el problema que representa el aprendizaje significativo de aquellos conceptos no puede considerarse resuelto (Furió y Guisasola, 2001; Rainson y Viennot, 1999; Psillos, 1998). 
Las investigaciones en enseñanza de las ciencias que tratan sobre las dificultades de aprendizaje en el estudio elemental de circuitos de corriente continua (Duit, 1993) han revelado que el concepto de diferencia de potencial es uno de los que presenta mayores problemas (Eylon y Ganiel, 1990; Steinberg, 1992). Este concepto está directamente relacionado con los de campo y potencial eléctricos que se enseñan en electrostática (Benseghir y Closset, 1996; Furió y Guisasola, 1998a) y con el concepto de fuerza electromotriz de una pila (Page, 1977). Sin embargo, existen muy pocos trabajos en el campo de la investigación didáctica que aborden el problema de la enseñanza-aprendizaje del concepto de fuerza electromotriz en un contexto de circuito eléctrico elemental o en general. Esta ausencia de trabajos llama la atención si consideramos el importante papel que juega el concepto de fuerza electromotriz en el marco explicativo del electromagnetismo, dentro de lo que se considera en términos lakatianos núcleo duro de la teoría (Lakatos, 1970).

De acuerdo con Arons (1970), la fuerza electromotriz ( $\mathrm{fem}$ ) es una propiedad de los generadores de energía eléctrica que mide el trabajo por unidad de carga para mantener el flujo de cargas en el circuito eléctrico y la pérdida simultánea de calor por efecto Joule. Por ejemplo, en el caso de una pila en un circuito eléctrico sencillo, la fuerza electromotriz representa la energía química ${ }^{1}$ convertida en eléctrica por unidad de carga que atraviesa cualquier sección del circuito. En la interpretación del funcionamiento de los circuitos eléctricos sencillos, la pila es en definitiva la causa que mantiene constante una diferencia de potencial entre los extremos del circuito. En la pila tienen lugar una serie de acciones eléctricas no electrostáticas a través de las cuales se suministra energía a la unidad de carga y esta energía se cuantifica mediante la magnitud «fuerza electromotriz» (Chabay y Sherwood, 2002). En definitiva, la fuerza electromotriz es una magnitud que cuantifica la energía cedida a la unidad de carga por el generador eléctrico. Así pues, la magnitud «fuerza electromotriz» se establece de forma muy específica en relación con los conceptos de carga, potencial, campo e intensidad de corriente, y se utiliza para explicar el comportamiento de los generadores eléctricos en los circuitos eléctricos sencillos o bien la fuerza electromotriz de inducción (Arons, 1970).

Este trabajo pretende contribuir a esclarecer el origen de las dificultades en el aprendizaje de este concepto, con el siguiente esquema: $a$ ) Establecer los indicadores de comprensión del concepto, es decir, cuáles son los atributos y características que definen la noción. $b$ ) Responder a preguntas como: ¿El concepto de fem pertenece al ámbito de la electrostática o a la electrocinética? ¿Qué nociones, de algún modo, son fronterizas con la de fuerza electromotriz y están implicadas en la comprensión de dicho concepto?

Consideramos, de acuerdo con Nerssesian (1995), que los conceptos no emergen milagrosamente sino que son el resultado de un proceso arduo de resolución de problemas y de contrastación rigurosa de hipótesis iniciales. En este sentido, creemos que la historia de la ciencia se muestra como un instrumento útil (Saltiel y Viennot, 1985; Wandersee, 1992; Gil, 1993) al señalar dónde estuvieron los problemas en la construcción de estos conceptos y qué barreras epistemológicas hubieron de superarse. A partir de aquí es posible inspirar y elaborar actividades de aprendizaje que pueden ser una ayuda estimable para mejorar de forma sensible el aprendizaje significativo del concepto de fem así como del resto de los conceptos fronterizos con el mismo.

Así pues, en este trabajo nos ocupamos de los problemas de aprendizaje del concepto de fuerza electromotiz en un nivel introductorio realizando los siguientes pasos: a) conocer las principales dificultades que se produjeron en el desarrollo del concepto a lo largo de la historia; b) establecer las principales características de un aprendizaje significativo del concepto de fuerza electromotriz; c) desarrollar un diseño experimental que nos permita indagar en las principales dificultades de aprendizaje de este concepto en estudiantes de último curso de carrera y recién licenciados en ciencias.

El diseño experimental se ha aplicado a estudiantes de último curso de ingeniería y recién licenciados en ciencias (físicos y químicos) que realizan el Curso de Aptitud Pedagógica para dirigir su futuro profesional como profesores de enseñanza secundaria. Pretendemos responder a preguntas como: ¿En qué medida los estudiantes de último curso o recién licenciados entienden significativamente el concepto de fem? ¿Cuáles son las principales dificultades? ¿Distinguen el concepto de fem y diferencia de potencial?

\section{OBSTÁCULOS EPISTEMOLÓGICOS OUE SE PRESENTARON EN EL DESARROLLO DEL CONCEPTO DE FUERZA ELECTROMOTRIZ A LO LARGO DE LA HISTORIA}

\section{El concepto de fuerza electromotriz, de Volta}

El término fuerza electromotriz fue acuñado por Volta. Las investigaciones (Pearce, 1962; Varney y Fhiser, 1980; Pancaldi, 1990) ponen de manifiesto que hacia 1797 se encuentran los antecedentes del término en los escritos de Volta, y claramente en 1801 (Figuier, 1887). Volta, ocupado en su polémica con Galvani acerca del origen de la electricidad, descubrió que, cuando dos piezas descargadas de metales diferentes se ponen en contacto, ya sea directamente o con la intervención de un electrolito, los dos metales llegan a cargarse y permanecen cargados a pesar del hecho de que hay un excelente camino conductor a través del cual las cargas podían fluir para neutralizarse entre sí (Aguilar, 2001; Pancaldi, 1990; Taton, 1988). Hay una clara violación de la electrostática en este hecho, ya que, según lo que se conocía de ella, las cargas opuestas no se pueden separar o, si lo hacen, vuelven a recombinarse.

El simple hecho de que cargas diferentes se muevan espontáneamente separándose, cuando dos metales diferentes se ponen en contacto, señala que la acción es motriz. Sus experiencias llevaron a Volta a tomar conciencia de la propiedad de tales contactos (convenientemente conectados para formar un circuito cerrado) como causa de corrientes eléctricas continuas y esto, casi con toda certeza, le llevó a aceptar, con mayor convencimiento, la naturaleza motriz de tal acción. 
Volta decía que una nueva clase de «fuerza» o capacidad actuaba sobre las cargas separándolas y manteniéndolas separadas y nombró la acción como fuerza electromotriz, nombre con el que se la conoce desde entonces. Las principales observaciones y medidas de Volta fueron, sin embargo, no de corrientes sino de la diferencia de potencial electrostático (que él llamaba entonces tensión eléctrica) que se producía entre los metales (con o sin la intervención de electrolitos) y que Volta medía con sus electroscopios. Estuvo claro para él que la «tensión» observable (d.d.p. en el actual marco conceptual) en la ausencia de corrientes debería contrarrestar la fuerza electromotriz que la causaba, de lo que concluyó que la «tensión» eléctrica observada, para el caso del circuito abierto, era una medida de la fuerza electromotriz, lo que sin duda era una hipótesis atrevida a tenor de las elaboraciones teóricas al respecto durante los cuarenta años siguientes. Como veremos a continuación, la hipótesis de Volta fue olvidada en un contexto donde el paradigma dominante era el newtoniano-coulombiano.

\section{El esquema conceptual dominante en la Francia de 1800: la interpretación electrostática de la pila}

En la primera década del siglo XIX la física experimental sufrió una transformación (Brown, 1969; Buchwald, 1977; Fox, 1990; Frankel, 1977; Guerlac, 1976; Sutton, 1981). Los esfuerzos de Laplace, Berthollet y sus discípulos en la Société d'Arcueil transformaron una exposición cualitativa de la ciencia en una disciplina rigurosamente matematizada. Esta transformación tiene sus raíces en los años 1780 y su culminación en el año 1810, pero el período crucial es entre 1800-1810 cuando el círculo de Arcueil se impone de manera consciente la tarea de interpretar matemáticamente la física general y experimental. Laplace y Berthollet, que habían sido amigos desde principios de 1785, eran activos propagandistas del movimiento para una nueva física y una nueva química. Berthollet escribió la introducción a las primeras memorias sobre la Société d'Arcueil en 1807. Allí se podía leer: «Para el progreso en la física y en la química es necesario alcanzar gran precisión en los hechos, se deben perfeccionar todos los significados de los hechos establecidos y comparar los resultados de los diferentes físicos [...] solamente a través de esta labor es posible llegar al establecimiento de teorías firmes $[. .] »$.

A pesar de que los físicos de esta época en Francia eran con creces los mejor organizados y los más sofisticados de Europa, es preciso dejar claro que, durante los primeros años de la centuria, las teorías no se desarrollaron de un modo continuo y acumulativo. En el caso de la pila y de la electricidad, en general las fuentes revelan que:

1) Al principio, los físicos hicieron de la pila, no del circuito, el centro de la investigación. Toman prestado de la electrostática técnicas instrumentales para estudiar la configuración electrostática de la pila; es más, los datos procedentes de las investigaciones de los fenómenos químicos realizadas por Humphry Davy y sus seguidores fueron explicados mediante detalladas analogías electrostáticas.

2) Después de 1820, a partir de las experiencias de Oersted y Ampère se desarrollaron otras posibilidades instrumentales y teóricas que hicieron del circuito en su conjunto el principal objeto de investigación, y la corriente eléctrica llegó a parecerse a lo que hoy entendemos por la corriente por primera vez. Si bien es preciso no olvidar que, durante más de los primeros cuarenta años del siglo XIX, se distinguía entre galvanismo y electricidad que era exclusivamente la electrostática ${ }^{2}$. Ello dio lugar a que durante este tiempo las explicaciones acerca de lo que hoy denominaríamos circuitos de corriente continua no se formularan.

Lo anterior no es de extrañar, ya que, en la corriente de pensamiento dominante, Coulomb, que gozaba de un enorme prestigio debido a su habilidad experimental y su precisión matemática, había ideado un mecanismo mediante el cual podía medir la carga de cualquier cuerpo. Antes de la entrada en escena de Volta, esto suponía cuantificar sobre cualquier aspecto experimental relacionado con la electricidad.

El descubrimiento de Volta dio lugar a numerosos estudios e informes. Las conclusiones más llamativas, desde nuestro punto de vista actual, bien podrían ser la distinción que se realizaba entre «electricidad ordinaria» (la obtenida por fenómenos electrostáticos) y «electricidad galvánica» (la obtenida por la pila). Los defensores de esta distinción argumentaban que: $a$ ) el shock galvánico es fuerte y continuo, los de la electricidad ordinaria son instantáneos; $b$ ) durante la conmoción galvánica no pueden detectarse signos de electricidad ordinaria con un electrómetro. En relación con este tipo de argumentaciones, decía un influyente científico de la época, Robertson: «Yo rechazo el fluido eléctrico como principio del galvanismo para atribuirlo a un ácido sui generi».

Las explicaciones que Volta elaboró para su descubrimiento no encajaban bien en los esquemas imperantes en la Francia napoleónica y sólo, debido a lo relevante del invento como a la fama y consideración de la que gozaba Volta ante el influyente Laplace, se creó una comisión que estudiase la llamada pila. Dicha comisión estuvo constituida por: Laplace, Coulomb, Fourcroy, Guyton y Biot. Este último actuó como portavoz siendo además el responsable de la redacción de las conclusiones del grupo de estudiosos.

Biot, en sus explicaciones como portavoz de la comisión mencionada, difería de Volta en dos aspectos sutiles pero cruciales: en la naturaleza de la fuerza electromotriz y en el significado de la circulación eléctrica. En palabras de este autor: «La corriente de Volta constituida por electricidad continuamente impelida se rompe en una multitud de instantáneas descargas de corriente y la pila ahora entrega un aparente shock continuo, cuya apariencia de continuidad depende de la circunstancia accidental por el hecho de que la pila repolariza antes los órganos del cuerpo descargando completamente la electricidad que habían recibido». Mediante estas sutiles alteraciones, Biot recogía la preocupación de la comisión con el aspecto teórico de la electrostática coulombiana (Brown, 1969).

En realidad, nos encontramos con dos teorías diferentes para explicar el fenómeno revolucionario de la pila, que 
cambió la forma de trabajar en electricidad y su posterior desarrollo. Para Biot y la Comisión, es decir, para el paradigma coulombiano dominante en la época, la fuerza electromotriz definida por Volta era la capacidad que tenían algunos cuerpos para generar electricidad en otros. Vemos que esta interpretación es radicalmente diferente de la original de Volta, que entiende la fuerza electromotriz como la capacidad para separar cargas y mantenerlas separadas, relacionándola con medidas de diferencia de potencial. Las explicaciones de Volta que no se ajustaban al paradigma electrostático vigente no encuentran, por tanto, ningún eco y caen en el olvido. Posteriormente el Tratado sobre Electricidad y Magnetismo, de Maxwell, tampoco relaciona a Volta con el concepto de fem, lo que definitivamente arrincona las aportaciones notorias del físico italiano.

Desde una visión de la naturaleza de la ciencia que concibe las teorías como procesos de argumentación donde los científicos relacionan conjeturas imaginativas con rigurosos procesos de contrastación (Giere, 1991; Toulmin, 1972), no resulta difícil comprender el largo y tortuoso camino que hubo de recorrerse hasta que se unificaron las explicaciones para los distintos experimentos de lo que terminaría por ser la electricidad y el magnetismo. Piénsese que hacia el primer tercio del siglo XIX estaban por resolver algunas cuestiones, necesarias para poder realizar estudios cuantitativos acerca de los circuitos eléctricos de manera satisfactoria (Humphreys, 1937), entre las que cabe destacar: $a$ ) la consecución de una corriente constante a lo largo de todo el circuito; $b$ ) la invención de los galvanómetros y la aplicación de la teoría magnética para relacionar convenientemente corrientes y efectos magnéticos; $c$ ) acuerdos sobre las implicaciones que podrían deducirse de la medida de tales efectos magnéticos; $d$ ) la medida en una unidad común para la resistencia de cada parte del circuito.

\section{La emergencia del paradigma energetista: la cons- trucción del concepto de fuerza electromotriz actual}

En la primera mitad del siglo XIX, los conceptos de carga, potencial, diferencia de potencial, capacidad, etc. pertenecían al ámbito de las explicaciones teóricas de la electricidad común o electrostática, que nada tenían que ver con el galvanismo o la magnetoelectricidad, ámbitos de experiencias en los que se recogían los fenómenos conocidos en relación con las pilas y la inducción respectivamente. Tampoco existía un modelo de la materia que permitiera unificar las explicaciones que se daban a los fenómenos eléctricos en general. En este sentido, cabe señalar que la noción de carga (término que introduce Franklin en el siglo anterior) nada tiene que ver con el concepto de carga actual. Para Franklin, la fricción «cargaba» al material en cuestión de fluido eléctrico, lo que daba lugar a que se manifestaran una serie de efectos. En el primer tercio del siglo XIX, el consenso en torno a la noción de carga no iba mucho más allá de la noción de Franklin. El concepto de carga, en la forma en la que hoy se concibe no estaría disponible hasta años después, en la época en la que Maxwell realiza sus contribuciones (Buchwald, 1985; Darrigol, 1998).

En el contexto descrito hay que entender las contribuciones realizadas por Ohm, cuyos trabajos de investigación trataban de relacionar la fuerza magnética ejercida por una corriente y la naturaleza del conductor que transportaba la corriente. Conviene dejar esto bien claro porque, ni remotamente, Ohm había pretendido establecer una relación entre la intensidad de corriente de un circuito y la diferencia de potencial entre sus extremos. Las razones son bien sencillas: ni existía la noción de corriente eléctrica, ni existía la noción de potencial aplicada al caso de los circuitos galvánicos. Ni que decir tiene que tampoco existía la idea de resistencia (Archibad, 1988; Brown, 1969; Humphreys, 1937; Pearce, 1962; Whittaker, 1951).

Lo que Ohm obtuvo en el transcurso de sus investigaciones fue una serie de resultados experimentales que permitieron la construcción de la primera teoría coherente de la conducción eléctrica. Ohm trató de establecer un modelo explicativo para los circuitos galvánicos (lo que hoy llamaríamos circuitos de corriente continua). En su libro El circuito galvánico investigado matemáticamente (Archiblad, 1988), Ohm definió su noción de fuerza electroscópica, antecedente inmediato del potencial para el caso de los circuitos eléctricos. Posteriormente, definió la tensión (en sus escritos utilizó el vocablo spannung) en una porción del circuito, como la diferencia entre las fuerzas electroscópicas en sus extremos.

El modelo de Ohm se encuentra situado en el paradigma electrostático basado en el modelo de conducción de calor establecido por Fourier en 1822. Del mismo modo que este autor distingue entre calor y temperatura como piedra angular de su propuesta, suponiendo que el flujo de calor entre dos partes adyacentes de un sistema es proporcional a sus temperaturas, la teoría de Ohm convierte la cantidad de electricidad en la variable crítica, haciendo descansar sobre la densidad superficial de carga (fuerza electroscópica) el mismo papel que matemáticamente jugaba la temperatura en la teoría de Fourier. La fuerza electroscópica se medía con un «instrumento electrostático» como un termómetro mide la temperatura (Archibald, 1988; Whittaker, 1951).

Cuando alrededor de 1847 Kichhoff aborda el estudio de las leyes de Ohm, el electromagnetismo está mucho más elaborado y las distancias entre electricidad y galvanismo se han reducido; existen suficientes semejanzas y el paradigma electrostático, en Alemania más aún, no es el que prevalece (Archibald, 1988). El físico alemán, tras el análisis de los trabajos de Ohm sobre la conducción y de Kohlrausch acerca de la medida de tensiones en condensadores, identifica la fuerza electroscópica de Ohm con la diferencia de potencial (Whittaker, 1951). Esta identificación sólo fue posible desde el cambio de paradigma que supuso la introducción del concepto de energía y, en concreto, la versión cuantitativa a través de las funciones de potencial de Poisson. Este nuevo paradigma energetista permite la interpretación global macroscópica de los circuitos eléctricos. El propio Helmholtz utilizó los trabajos de Kirchhoff en sus elaboraciones últimas acerca del principio de conservación de la energía, publicado en 1847.

El paradigma energetista emergente recibiría nuevos impulsos con la teoría de campo iniciada por Faraday y 
fundamentada posteriormente por Maxwell en 1865. La noción de campo permite elaborar un marco teórico desde el que explicar los resultados de la experimentación en parcelas como el calor, la electrostática, el magnetismo, la luz, etc. (Popper, 1985). Este marco conceptual permite desarrollar el concepto de energía asociada al campo, ya sea éste conservativo (energía potencial) o no conservativo (fuerza electromotriz en el caso de la pila en circuitos sencillos de corriente continua y en fenómenos de inducción electromagnética). Es en este paradigma energético y de campo donde actualmente definimos los conceptos de potencial eléctrico y fuerza electromotriz; dos conceptos epistemológicamente relacionados pero distintos, al igual que ocurre con otros conceptos como, por ejemplo, velocidad y aceleración en cinemática.

En el siguiente apartado nos ocupamos con más concreción de la definición actual de fuerza electromotriz dentro del paradigma energetista.

\section{INDICADORES DE UN APRENDIZAJE COM- PRENSIVO DEL CONCEPTO DE FUERZA ELECTROMOTRIZ}

De acuerdo con las dificultades detectadas en el análisis histórico y el marco teórico actual de la física, podemos establecer una serie de requisitos necesarios para un aprendizaje comprensivo del concepto de fuerza electromotriz y de sus relaciones con otros conceptos fronterizos como los de diferencia de potencial, carga e intensidad de corriente. Así pues establecemos como indicadores de su comprensión los siguientes:

1) Saber que la «fuerza electromotriz» (fem) es una propiedad de los generadores de energía eléctrica y no es una propiedad ni del circuito ni de las cargas. En el caso de circuitos eléctricos sencillos, es decir, constituidos por una pila, cables de conducción y resistencias (Fig. 1), la fem mide la energía que la pila da a la unidad de carga que atraviesa cualquier sección del circuito. Este tipo de proceso realizado en la pila suele consistir en una serie de reacciones químicas que de manera general podemos llamar acciones no conservativas.

Figura 1

La parte en trazo más grueso representa la parte del circuito constituido por la pila. La parte dibujada en trazo más fino representa el resto del circuito.

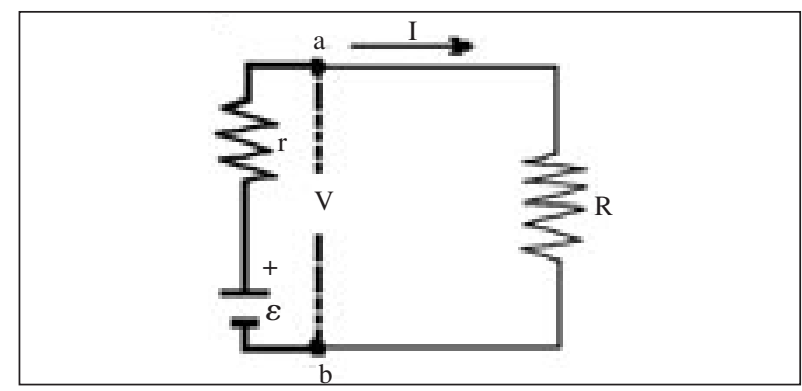

2) Lo anterior se concreta en saber que la fuerza electromotriz, en el caso de la pila, es la causa de una separación de cargas de distinto signo entre sus electrodos y, por tanto, la causa de una diferencia de potencial constante entre los mismos (puntos $a$ y $b$ del circuito de la figura 1). Este proceso de separación de cargas lo podemos explicar mediante una analogía entre la pila y el generador de Van der Graaf (Fig. 2). Al cabo de un cierto tiempo se ha establecido una diferencia de potencial entre los puntos A y B. Evidentemente la carga acumulada en la esfera A aumenta constantemente y, cuanto mayor sea la cantidad, mayor será la diferencia de potencial y más difícil será llevar las cargas de B a A, debido a la repulsión electrostática entre cargas del mismo signo. Así pues, sobre las cargas actúan dos tipos de fuerzas: las electrostáticas que tienden a repeler las cargas que llegan a la esfera A (fuerzas eléctricas y conservativas) y la fuerza de tipo mecánico (no conservativa) de la cinta transportadora que hace que las cargas llegen a la esfera A. El trabajo (por unidad de carga) realizado por estas fuerzas para separar las cargas y desplazarlas es la fuerza electromotiz.

Figura 2

Esquema del generador de Van der Graaf. En este generador la fricción de una cinta de material aislante en movimiento, con una escobilla B situada en la parte inferior, hace que la escobilla B quede cargada negativamente y la cinta positivamente.

Las cargas positivas son transportadas por la propia cinta y liberadas mediante una segunda escobilla,

sobre una esfera metálica hueca A situada en la parte superior. 
Del mismo modo que en la analogía descrita, en el interior de la pila, sobre las cargas, actúan fuerzas de naturaleza distinta: fuerzas no electrostáticas (acciones no conservativas) y fuerzas de repulsión electrostáticas (acciones conservativas). Así pues, la fuerza total se puede indicar como:

$$
\vec{F}_{\text {total }}=\vec{F}_{\text {elec. conservativa }}+\vec{F}_{\text {no elec. no conservativa }}=q \vec{E}+q \vec{E},
$$

...donde E es el campo eléctrico conservativo producido entre los extremos A y B del interior de la pila o del generador de Van der Graaf y E' es un campo no conservativo debido a las acciones no conservativas como, por ejemplo, reacciones químicas dentro de la pila. Ahora se puede comprender por qué Volta no profundizó en su concepto de fuerza electroscópica. El concepto de campo no formaba parte de las descripciones de la época y aparecería algunos años después con Faraday.

Si aplicamos la definición de fem como el trabajo necesario sobre la unidad de carga para describir una línea cerrada (circuito), obtenemos la definición operativa de fuerza electromotriz:

$$
f e m=\oint(\vec{E}+\vec{E}) \overrightarrow{d l}
$$

Como el campo E es conservativo, su integral, a lo largo de todo el circuito (una línea cerrada) es cero, y como el campo E' corresponde sólo a las acciones no conservativas dentro de la pila se definirá entre los extremos $a \mathrm{y}$ b. Así pues:

$$
f_{e m}=\int_{a}^{b} \vec{E}, \overrightarrow{d l}
$$

En conclusión, la fuerza electromotriz es una magnitud que cuantifica una transferencia de energía (de la pila a las cargas del circuito) asociada a un campo no conservativo.

3) El punto anterior implica saber distinguir entre la medida de la fuerza electromotriz de una pila y la medida de la diferencia de potencial entre los electrodos de una pila. Por ejemplo, en el caso del circuito de la figura 1, la fuerza electromotriz procede del hecho de que la pila separa cargas y crea una diferencia de potencial. La medida del trabajo realizado viene dada por la definición operativa de la ecuación (1) a nivel microscópico y a nivel macroscópico se da en función del potencial y de la intensidad de corriente como: $\varepsilon=\Delta V$ - Ir. Sin embargo, la diferencia de potencial que medimos entre los puntos $a$ y $b$ pertenece a la parte exterior del circuito (en trazo fino) y se corresponde con el trabajo realizado al mover la unidad de carga dentro de un campo eléctrico conservativo situado dentro del cable (Arons, 1970; Reitz, Milford y Christ, 1996; Chabay y Sherwood, 2002). En este sentido, la definición operativa de la diferencia de potencial viene dada a nivel microscópico como:

$$
V_{a b}=-\int_{a}^{b} \vec{E}, \overrightarrow{d l} \quad \text { y a nivel macroscópico como : } \mathrm{V}_{\mathrm{ab}}=\mathrm{I} \mathrm{R}
$$

En resumen, la diferencia entre la fuerza electromotriz y la diferencia de potencial viene dada por la medida de dos tipos diferentes de acciones producidas por factores radicalmente diferentes. La primera debida a un campo no conservativo y la segunda a uno conservativo (Esquema 1).

Conviene recordar que la diferencia entre un campo conservativo y uno no conservativo no es algo que se pueda «reducir»a diferentes ecuaciones matemáticas, tal y como lo demuestra la historia del tortuoso y complicado camino que diferentes grupos de investigación dirigidos por eminentes científicos tuvieron que recorrer para establecer el concepto actual de fuerza electromotriz.

Esquema 1

Relaciones y diferencias entre la fuerza electromotriz y la diferencia de potencial electrostático.

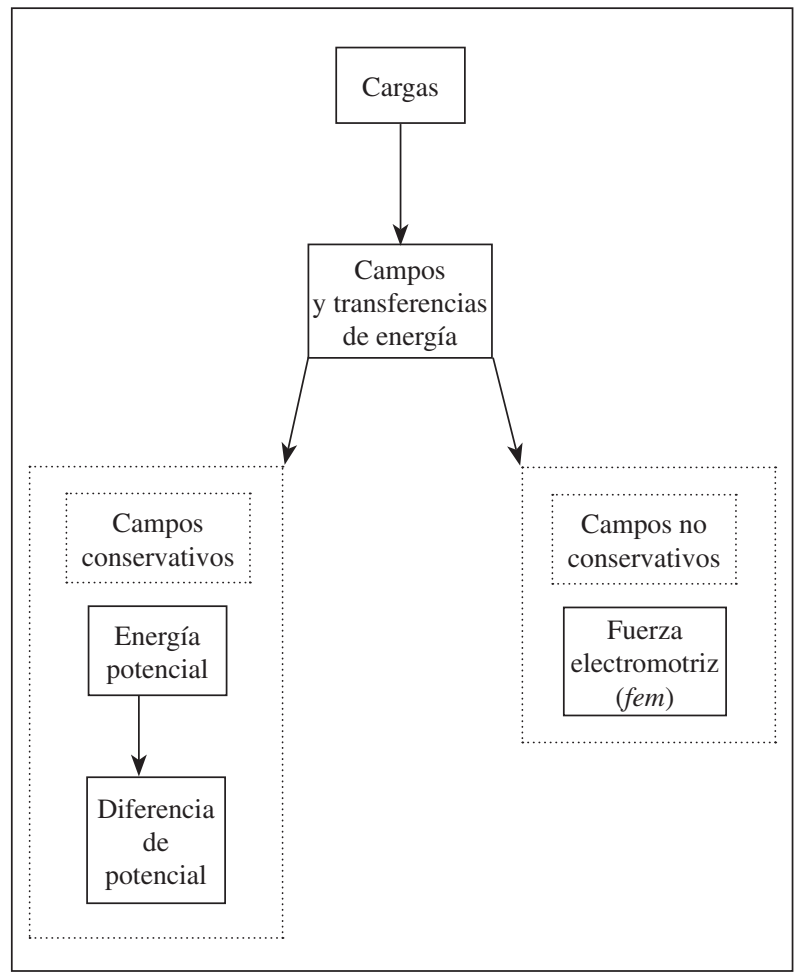

Hay que destacar que la investigación didáctica ha mostrado que todos los conceptos implicados en el esquema anterior son conflictivos en el proceso de enseñanza-aprendizaje (Hierrezuelo y Montero, 1991; Furió y Guisasola, 1998b, 2003; Martín, 1999; Martín y Solbes, 2001).

4) Saber que, en el caso de la inducción electromagnética, los campo eléctricos producidos no están relacionados con cargas, sino con flujo magnético variable $\mathrm{y}$, por tanto, no son conservativos, por lo que el trabajo realizado, al desplazar la unidad de carga por el circuito, lo mide la fuerza electromotriz y, en ningún caso, la diferencia de potencial (Lanzara y Zangara, 1995). 


\section{DISEÑO EXPERIMENTAL Y EMISIÓN DE HI- PÓTESIS}

La revisión histórica realizada hasta aquí nos ha permitido observar los problemas y las dificultades que tuvo que afrontar la comunidad científica y cuya resolución permitió ir construyendo el modelo explicativo de la circulación de corriente por un circuito eléctrico sencillo y la construcción del concepto de fuerza electromotiz. En este sentido, se puede asumir, a título de hipótesis, que estas dificultades ontológicas y epistemológicas podrían constituir dificultades de aprendizaje en la interpretación científica de circuitos sencillos de corriente continua y, en concreto, del concepto de fuerza electromotiz, y que, consecuentemente, las tendrán los estudiantes de último curso de universidad y los recién licenciados que orientan su formación para ser futuros profesores, los cuales muestran un aprendizaje más bien memorístico y operativista del concepto de fuerza electromotiz, poco comprensivo y no diferenciado del concepto de diferencia de potencial. Esta hipótesis de trabajo la concretamos en los siguientes enunciados:

1) Los futuros profesores de ciencias no diferencian entre las propiedades de las cargas y las propiedades del campo y del circuito. Identificarán el potencial y la diferencia de potencial como propiedad de las cargas.

2) Los futuros profesores de ciencias no contemplan la fem como una propiedad característica de la pila, por lo que no distinguirán entre la medida de la fem de una pila y la medida de la diferencia de potencial entre los electrodos de la pila.

3) Presentarán confusión al explicar el concepto de diferencia de potencial en el contexto de un circuito sencillo de corriente continua.

Para contrastar las hipótesis anteriores se ha diseñado un cuestionario en forma de preguntas de opción múltiple y un guión de entrevista con las mismas preguntas (Anexo). Se trata de averiguar no sólo cuál es la idea de los futuros profesores acerca del concepto fuerza electromotiz sino también indagar sobre qué relaciones establecen entre este concepto y otros cercanos a él como diferencia de potencial, carga eléctrica, campo e intensidad de corriente. Para averiguar si los futuros profesores diferencian entre las propiedades de las cargas y del circuito (campo, pila, etc.) se ha diseñado la primera cuestión. En ella se atribuyen a las cargas propiedades que no poseen como fuerza electromotriz o diferencia de potencial. Para profundizar en el aprendizaje comprensivo del concepto fuerza electromotriz se han diseñado las cuestiones 2, 4 y 5 . En ellas se pregunta a los estudiantes sobre fuerza electromotiz como propiedad de la pila (cuestiones 2 y 4) o de acciones no conservativas (cuestión 5) y su relación con la magnitud «diferencia de potencial». Para averiguar si existe confusión en el significado del concepto diferencia de potencial que está muy relacionado con el de fuerza electromotriz, se ha diseñado la cuestión 3. Estas mismas cuestiones se han preguntado en el guión de las entrevistas.
Hemos utilizado una combinación de métodos cuantitativos (cuestionario) y cualitativos (entrevistas), ya que consideramos que la detección de concepciones de los estudiantes abarca múltiples aspectos que deben ser detectados con una variedad de métodos. Además la utilización de ambos métodos para la consecución de un mismo objetivo pueden ser complementarios y ofrecernos perspectivas que no ofrecerían ninguno de los dos por separado (Cook y Reinardt, 1982).

El cuestionario se ha pasado a un grupo de 51 estudiantes de último curso de ingeniería industrial y recién licenciados físicos y químicos, que realizaban el CAP para ser futuros profesores de ciencias experimentales. Al pasar la encuesta se les indicó que iban a colaborar en una investigación sobre dificultades en el aprendizaje y se les solicitó poner su nombre y teléfono a fin de pedirles aclaraciones posteriores.

Se realizaron nueve entrevistas. En todos los casos se procedió de la misma manera, se les presentaban sus respuestas y se les invitaba a completarlas, mejorarlas o ratificarlas. Dado el caso, el entrevistador tomaba notas que luego leía a los entrevistados para que éstos ratificaran una vez más si se recogían adecuadamente sus ideas.

Nuestras previsiones eran que, tanto por el nivel de los estudios cursados como por estar próximo el final de los estudios de licenciatura, sus conocimientos fuesen recientes y, por tanto, no deberían presentar errores relevantes en las aplicaciones de conceptos básicos del electromagnetismo.

\section{RESULTADOS Y ANÁLISIS DE LOS MISMOS}

Conviene aclarar, desde el principio, que los datos que se recogen en las tablas se corresponden con el número de elecciones que tiene una determinada opción; otra cosa es que la elección, aun en caso de ser la correcta, esté bien justificada. Creemos que en muchos casos, la mera elección es ya sintomática del nivel del aprendizaje; por ejemplo, si un determinado número de alumnos dice que el concepto de potencial utilizado en el estudio de los circuitos es diferente al concepto de potencial que se utiliza en electrostática, pensamos que, al margen de la justificación que se ponga en juego, la elección es en sí indicativa del pobre aprendizaje del conocimiento en cuestión. Para el análisis de las respuestas del cuestionario y de las transcripciones de las entrevistas, se ha seguido una metodología estándar como la indicada por Cook y Reinardt (1982).

En la primera cuestión, la respuesta correcta académicamente es la $d$, ya que las cargas poseen energía potencial (en virtud de su configuración) o cinética. En este caso, lo que varía es la energía potencial de las cargas, pero, en ningún caso, las magnitudes que se mencionan, ya que son propias de otros elementos del circuito. Los resultados obtenidos se encuentran en la tabla I. 
Tabla I

Resultados de la cuestión 1.

\begin{tabular}{|c|c|}
\hline Cuestión 1 & $\begin{array}{c}\text { CAP }(\mathbf{N}=\mathbf{5 1}) \\
\text { Número (porcentaje) }\end{array}$ \\
\hline Opción a & $1(2 \%)$ \\
\hline Opción b & $8(16 \%)$ \\
\hline Opción c & $28(55 \%)$ \\
\hline Opción d & $8(16 \%)$ \\
\hline NC & $6(11 \%)$ \\
\hline
\end{tabular}

El primer resultado llamativo es que más de la mitad de los estudiantes escogen la opción $c$, que es incorrecta. Estos resultados se vieron confirmados en las entrevistas que posteriormente se mantuvieron. A continuación exponemos un ejemplo que es ilustrativo del tipo de respuesta de la opción $c$ :

Cuando una carga se desplaza de un electrodo de una pila a otro, está pasando de un potencial menor a otro mayor; por tanto, varía su potencial. Si bien habitualmente se considera que las cargas van del electrodo positivo al negativo, eso es un convenio utilizado.

Llama también la atención que de los 7 estudiantes que escogen la opción correcta $(d)$, sólo uno la justifica más o menos correctamente y otro la señala pero no la justifica. Un ejemplo del tipo de justificaciones excesivamente ambiguas es el siguiente:

No varía nada, sólo hay un desplazamiento de electrones.

La única respuesta cuya justificación puede considerarse aproximadamente correcta es la siguiente:

Es la $d$. Si consideramos la carga: ésta al moverse pasa a regiones de menor a mayor potencial dependiendo de su carga positiva o negativa. Pero, propiamente hablando, la carga no tiene ninguna de estas magnitudes; por ejemplo, no se puede decir concretamente el potencial de una carga sino más bien el potencial al que está sometida una carga.

En la cuestión 2, la opción correcta académicamente es la $b$, ya que, al ser «la fuerza electromotriz» una característica de la pila y al mantenerse ésta, también se mantiene la magnitud «fuerza electromotriz». En el circuito propuesto se cumple que $\varepsilon=\mathrm{V}+\mathrm{Ir}$, donde $r$ es la resistencia interna de la pila. Si para una misma pila variamos los elementos del circuito, variará la intensidad y también la diferencia de potencial entre sus extremos. Los resultados se indican en la tabla II.

Tabla II

Resultados de la cuestión 2.

\begin{tabular}{|c|c|}
\hline Cuestión 2 & $\begin{array}{c}\text { CAP }(\mathbf{N}=\mathbf{5 1}) \\
\text { Número (porcentaje) }\end{array}$ \\
\hline Opción a & $18(35 \%)$ \\
\hline Opción b & $20(39 \%)$ \\
\hline Opción c & $0(0 \%)$ \\
\hline Opción d & $1(2 \%)$ \\
\hline NC & $8(16 \%)$ \\
\hline Otras $^{1}$ & $4(8 \%)$ \\
\hline
\end{tabular}

${ }^{1}$ En la casilla «Otras» se incluyen los alumnos que han escogido dos o más opciones.
Sólo el 39\% de las opciones escogidas son correctas, pero las justificaciones de la opción elegida no son del todo correctas o son claramente deficientes. Así, sólo cinco estudiantes se refieren textualmente a la fem como una propiedad característica de la pila; los demás hacen referencia a definiciones memorísticas sin significado.

La otra opción mayoritaria es la $a$ elegida por el 35\% de los estudiantes. Ejemplo de razonamientos utilizados por los estudiantes que eligen esta categoría serían los siguientes:

La diferencia de potencial entre los bornes, ya que tanto la intensidad como la fuerza electromotriz van a variar en función de los elementos (resistencia, etc.) propios del circuito.

Todas son correctas (menos la $d$ ), pues una pila es, por definición, un dispositivo que mantiene constante la d.d.p. existente entre los bornes. Su fuerza electromotriz es también constante y también constante la intensidad de corriente que «circula» por su interior.

En estas explicaciones se observa que centran el análisis en un visión incorrecta de la diferencia de potencial entre los bornes de una pila y no dan ninguna explicación sobre lo que sucede con la fuerza electromotriz. Parece como si esta magnitud no desarrollara ninguna función y careciera de significado para ellos o se identificara con la diferencia de potencial.

En la tercera cuestión, sabemos que, para que circule corriente, es necesaria una diferencia de potencial entre los extremos de un conductor. Así pues, la respuesta correcta es la $b$. Las respuestas de los estudiantes nos pueden indicar la idea que tienen sobre el concepto de diferencia de potencial y su aplicación en un contexto electrocinético. Los resultados obtenidos se indican en la tabla III.

Tabla III

Resultados de la cuestión 3 .

\begin{tabular}{|c|c|}
\hline Cuestión 3 & $\begin{array}{c}\text { CAP }(\mathbf{N}=\mathbf{5 1}) \\
\text { Número (porcentaje) }\end{array}$ \\
\hline Opción a & $1(2 \%)$ \\
\hline Opción b & $22(43 \%)$ \\
\hline Opción c & $5(10 \%)$ \\
\hline Opción d & $11(21 \%)$ \\
\hline NC & $12(24 \%)$ \\
\hline
\end{tabular}

La opción correcta $(b)$ es seleccionada por menos de la mitad de los estudiantes (44\%) y sólo la justifican correctamente 6. Veamos algunos ejemplos de justificaciones incorrectas de los estudiantes:

A pesar del movimiento se puede tratar el problema como dos concentraciones de carga (en los bornes de la pila), eso sí, teniendo en cuenta la variación de magnitud en cada uno de los bornes debido al movimiento de las cargas. 
Potencial electrostático es el que se produce como consecuencia de atracción de cargas: $\mathrm{F}=\mathrm{k}$ e$\cdot e=/ \mathrm{r}^{2}$. Si las cargas están en movimiento, crean una energía y ésta es la responsable de la diferencia de potencial.

Parece que existe una confusión a la hora de definir correctamente el concepto de diferencia de potencial y su función en un circuito. Esta impresión se confirma al constatar que el $22 \%$ de los futuros profesores elige la opción $d$. En sus explicaciones admiten que no se trata de la misma magnitud que se emplea en electrostática; las justificaciones también ilustran un aprendizaje memorístico y poco riguroso. Las siguientes son algunos ejemplos:

No se trata del mismo concepto. En electrostática, el potencial simboliza la energía potencial por unidad de carga que existe en un cierto punto de un campo eléctrico. Por contra, en un circuito se usa el concepto de d.d.p. entre dos puntos como condición indispensable para que una carga eléctrica se desplace entre dos puntos.

«En electrostática hablamos de potencial de una carga y en circuitos eléctricos hablamos de diferencias de potencial entre dos puntos. Así: $\mathrm{V}=\mathrm{I} \quad \mathrm{R}$ (circuito); $\mathrm{V}=\left(1 / 4 \Pi \varepsilon_{0}\right) \mathrm{q} / \mathrm{r}$ (Electrostática) (Estudiante del CAP).

El siguiente es un fragmento de la entrevista que mantuvimos posteriormente con esta misma estudiante:

Entrevistador: ¿Quieres añadir algún matiz a tu respuesta a esta pregunta?

Estudiante: Bueno... yo creo que está muy claro... Basta con interpretar las dos ecuaciones matemáticas.

Entrevistador: ¿Te importaría interpretarlas?

Estudiante: Bueno... yo creo que es evidente... los segundos miembros de esas ecuaciones matemáticas no son los mismos, luego no puede tratarse de la misma magnitud V.

Entrevistador: ¿Por qué emplear entonces la misma letra y el mismo nombre para magnitudes diferentes?

Estudiante: No sé... puede tratarse de un convenio... no sería la primera vez... ¿no?

En la cuestión 4 se vuelve a incidir sobre el significado de la fuerza electromotriz y la diferencia de potencial en un circuito eléctrico sencillo. En este caso sabemos que, si dos puntos a diferente potencial se conectan mediante un conductor, pasarán las cargas entre esos dos puntos y al cabo de muy poco tiempo el potencial acabará siendo el mismo y cesará el paso de cargas. Precisamente, en un circuito, la función de la pila es transferir energía para mantener la diferencia de potencial entre esos dos puntos. El proceso contiene «acciones no conservativas» (producidas por la pila) y se describe mediante la magnitud «fuerza electromotriz»; por tanto, la ecuación correcta es la $b$. La ecuación de esta opción nos indica, de acuerdo con el principio de conservación de la energía, que la energía transformada al pasar corriente por una resistencia necesita de una fuente de energía y que la energía transferida por la fuente se mide mediante la fuerza electromotriz. Los resultados obtenidos se muestran en la tabla IV.
Tabla IV

Resultados de la cuestión 4.

\begin{tabular}{|c|c|}
\hline Cuestión 4 & $\begin{array}{c}\text { CAP (N= 51) } \\
\text { Número (porcentaje) }\end{array}$ \\
\hline Opción a & $16(31 \%)$ \\
\hline Opción b & $7(14 \%)$ \\
\hline Opción c & $19(37 \%)$ \\
\hline Opción d & $1(2 \%)$ \\
\hline $\mathrm{NC}$ & $3(6 \%)$ \\
\hline $\mathrm{NJ}^{1}$ & $5(10 \%)$ \\
\hline
\end{tabular}

${ }^{1}$ Se recogen aquí los alumnos/as que señalan una opción pero no la justifican.

En la gran mayoría de las explicaciones, los estudiantes coinciden en presentar confusiones entre la fuerza electromotriz y la diferencia de potencial y en identificar las expresiones de $\mathrm{V}=\mathrm{IR}$ y $\xi=\mathrm{IR}$ como expresiones de la ley de Ohm (el 37\% lo hace explícitamente en la opción $c$. También, cuando eligen la opción correcta, razonan erróneamente, como podemos ver en los siguientes ejemplos:

La $b$, porque la caída de potencial a través de la pila es $\xi$. Si consideramos la caída de potencial entre los mismos puntos, pero a través de la resistencia, ésta será $V_{R}=I R$. Como ambas deben ser iguales, entonces $\xi=\mathrm{V}_{\mathrm{R}}=\mathrm{IR}$.

La $b$, porque $\mathrm{E}=\mathrm{IR}$ es la expresión que corresponde a la ley de $\mathrm{Ohm}$, y de aplicación en el análisis de circuitos eléctricos. En circuitos eléctricos no tendría sentido hablar de d.d.p. electrostático, ya que éste es un concepto relativo a campos eléctricos. Es decir, no depende de la d.d.p. en un campo eléctrico del flujo de cargas eléctrico I, ni de R, sino del campo eléctrico.

El 32\% de los estudiantes elije la opción a como correcta y razona de forma semejante, imputando a la necesidad de que se cumpla la ley de Ohm, el que se cumpla la relación $\mathrm{V}=\mathrm{IR}$. Veamos un ejemplo que ilustra tipo de razonamientos de los estudiantes que elijen esta opción:

La respuesta correcta es la $a$ siempre y cuando el interruptor permita que el circuito esté cerrado. Es la a porque es la expresión de la ley de

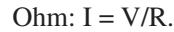

Para recabar más información acerca de la confusión que presentan los estudiantes en las explicaciones al identificar las ecuaciones $\mathrm{V}=\mathrm{IR}$ y $\xi=\mathrm{IR}$ como expresiones de la ley de Ohm, en las entrevistas se preguntó al respecto. Veamos a continuación una respuesta estándar:

Entrevistador: ¿Las ecuaciones V $=\mathrm{IR}$ y $\xi=\mathrm{IR}$ son expresiones equivalentes de la ley de Ohm?

Alumno: Bueno... sí... depende del caso...

Entrevistador: En este caso, claro. Luego nos podemos referir a otras situaciones. (Señalando el dibujo de la pregunta.)

Alumno: En este caso sí porque... la resistencia interna de la pila es nula y entonces las medidas entre los bornes de la pila y entre la resistencia es la misma, ya que, si la intensidad que circula por el circuito es I y la caída de tensión es la misma tanto en la pila como en la resistencia... tiene que ser la misma... entonces se debe cumplir el apartado $c \ldots$ De 
todas formas yo creo que... bueno la fem y al d.d.p. son la misma... cosa... miden la misma cosa..

Entrevistador: ¿Qué miden?

Alumno: Tensión.

En la pregunta 5 de nuevo se trata de indagar si los estudiantes distinguen entre la magnitud «fuerza electromotriz», la cual mide intercambios de energía que incluyen «acciones no conservativas», y la magnitud «diferencia de potencial», que sólo mide acciones producidas por (en el seno de) campos eléctricos conservativos. El fenómeno que se presenta es una inducción electromagnética producida por un campo magnético variable; por tanto, la acción es no conservativa y su medida se realizará a través de la magnitud «fuerza electromotriz». En esta situación no tiene sentido hablar de intensidad de corriente producida por una diferencia de potencial. Así pues, las opciones 1 y 4 son correctas desde el punto de vista académico y la 2 y 3 falsas. La tabla $V$ recoge los resultados en términos de número de encuestados que señala y justifica un enunciado de forma correcta/incorrecta o no contesta.

Tabla V

Resultados de la cuestión 5.

\begin{tabular}{|c|c|c|c|}
\hline & Verdadero & Falso & NC \\
\hline Cuestión 5 & CAP & CAP & CAP \\
\hline Enunciado 1 & $16(31 \%)$ & $29(57 \%)$ & $6(12 \%)$ \\
\hline Enunciado 2 & $7(14 \%)$ & $29(57 \%)$ & $15(30 \%)$ \\
\hline Enunciado 3 & $18(35 \%)$ & $6(12 \%)$ & $27(53 \%)$ \\
\hline Enunciado 4 & $7(14 \%)$ & $37(72 \%)$ & $7(14 \%)$ \\
\hline
\end{tabular}

Respecto al primer enunciado, la mayoría de los estudiantes $(57 \%)$ responde de forma incorrecta (el 69\% del total si entendemos como no correcto no contestar). En las entrevistas mantenidas sobre esta cuestión se detectó que el problema se presentaba con la segunda parte del enunciado «pero no se produce una diferencia de potencial electrostático entre los puntos A y $\mathrm{B} »$. Los estudiantes afirmaban que, si hay corriente, hay diferencia de potencial o que, si hay fuerza electromotriz, hay diferencia de potencial, haciendo la analogía con un circuito con pila. Como veremos, estos razonamientos son coherentes con los que se utilizaron en los enunciados posteriores.

Aproximadamente un tercio de los estudiantes elige la opción correcta en el enunciado 1, pero sólo dos han justificado correctamente la elección. Veamos la respuesta de uno de ello:

Como el campo magnético es variable, se induce una fuerza electromotriz de acuerdo con la ley de Faraday. Se cumple:

$$
\oint B \cdot d l=-\frac{d}{d t} \int_{s} B \cdot d S
$$

Es decir, se induce una fem. El potencial electrostático es debido a un campo conservativo y este campo inducido no lo es; por tanto, no existe diferencia de potencial electrostático entre A y B.

Respecto a explicaciones incorrectas dentro de considerar el enunciado como correcto, se podría decir que los estudiantes identifican el resultado como una situación correcta posible, en relación con la inducción magnética, por eso señalan una opción como verdadera, pero, cuando argumentan sobre la veracidad del enunciado, entran en contradicciones y ponen al descubierto errores. Por ejemplo, la siguiente es la respuesta de un alumno:

Verdadero. Aparece una fem debido a la variación del campo magnético, pero debido a la continuidad del material conductor no hay diferencia de potencial entre A y B, no hay nada que impida la circulación de las cargas.

En el segundo enunciado es de destacar que casi un tercio de los estudiantes no contesta, lo que confirmaría el poco significado que tienen para los estudiantes las magnitudes implicadas en el enunciado. De los que contestan, la mayoría (57\%) escoge la opción correcta. Sin embargo, las justificaciones de casi todos incluyen una premisa errónea, ya que indican que «hay diferencia de potencial entre los puntos A y $\mathrm{B} »$. Este resultado sería coherente con el obtenido para el enunciado 1, ya que parece que los estudiantes opinan que no puede haber corriente en un circuito sin diferencia de potencial o bien no diferencian suficientemente fuerza electromotriz y diferencia de potencial para distinguir cuando existe una $u$ otra. Veamos un ejemplo de este tipo de respuestas:

Un campo magnético variable genera una fem en el conductor, que hace que la corriente se mueva por el anillo, pero produce una diferencia de potencial electrostática.

En el enunciado 3 sorprende de nuevo el elevado número de estudiantes $(53 \%)$ que no contesta a la pregunta o que responde verdadero o falso pero sin argumentar en absoluto. Esto podría indicar la falta de significado que tienen para los estudiantes las magnitudes de «fuerza electromotriz»y «diferencia de potencial». De los que lo hacen, la mayoría $(35 \%)$ encuentra el enunciado correcto. De nuevo se constata que la mayoría de los estudiantes utiliza el argumento de que, en general, no puede existir corriente sin diferencia de potencial.

En el enunciado 4 sólo una alumna responde con corrección reconociendo expresamente que no se trata de una configuración de campos conservativos y explica que la afirmación es verdadera, pero no lo argumenta. Se puede entender que en coherencia con lo dicho no hay diferencia de potencial electrostático y, por tanto, el voltímetro no lo podrá medir, pero el caso es que no lo razona, con lo que también parece como si optase por no pronunciarse explícitamente sobre lo que el voltímetro puede medir en una situación como ésta.

La mayoría de los estudiantes no se pronuncia sobre el tema o responde verdadero o falso sin justificar nada y sin que antes haya habido corrección o coherencia en sus razonamientos a los apartados anteriores que permitan valorar como justificada la opción de no responder. Todo 
ello permite pensar, junto con algunas otras respuestas, que se confirmaron en las entrevistas, que los alumnos no saben qué mide y cuál es el campo de validez de la aplicación del voltímetro.

\section{CONCLUSIONES E IMPLICACIONES DIDÁC- TICAS}

Los resultados obtenidos parecen confirmar que los estudiantes, al acabar sus estudios de licenciatura, no han logrado un claro significado de la fuerza electromotriz $\mathrm{y}$, lógicamente, presentan confusiones entre ésta y la diferencia de potencial. Así, no reconocen que la fuerza electromotriz es una característica de la pila (cuestiones 2 y 4) y, en consecuencia, la suelen atribuir a propiedades de las cargas (cuestión 1). En la situación particular de un fenómeno sencillo de inducción electromagnética (cuestión 5), casi la totalidad de los estudiantes presenta serias deficiencias en los significados de la fuerza electromotriz y diferencia de potencial. Así mismo, es llamativo que la gran mayoría de estudiantes asocie el paso de corriente sólo con la diferencia de potencial.

El número de alumnos que piensa que el concepto de potencial, en electrostática y en circuitos, es distinto (cuestiones 3 y 5) es significativo y pone de manifiesto que, en la enseñanza, si se dan por supuestos muchos aprendizajes, lo más probable sea que éstos no se produzcan o lo hagan de forma poco significativa.

A partir de estos resultados se abren nuevos interrogantes e implicaciones didácticas que pueden ayudar a cambiar las formas habituales en que se enseña el concepto de fuerza electromotriz. En efecto, que los estudiantes de último curso de universidad o los recién licenciados no diferencien entre fuerza electromotriz y diferencia de potencial puede ser una consecuencia lógica que se deriva de la falta de tratamiento específico de aquella magnitud en la enseñanza de la electricidad. Es de destacar que el concepto de fuerza electromotriz (fem) es pobremente definido y deficientemente explicado, incluso en libros de texto universitarios bien conocidos y ampliamente utilizados por generaciones de estudiantes. Esta deficiencia contrasta con la normal meticulosidad de la física al definir los términos y elucidar los conceptos (Page, 1977).

Surge, pues, una primera cuestión didáctica: ¿Por qué no se presta la suficiente atención a la magnitud «fuerza electromotriz»? En nuestra opinión, la hipótesis más plausible, que trataremos de fundamentar, va en el sentido de que el profesorado no ve la necesidad lógica de introducir este concepto a través de algunos de los siguientes argumentos: $a$ ) la fuerza electromotriz es sinónimo de diferencia de potencial en circuitos de corriente continua; $b$ ) el marco de justificación del concepto de fuerza electromotriz es energético (balance energético del conjunto completo del circuito); y exige explicaciones en campos no conservativos diferenciándolos del campo eléctrico conservativo en el interior del cable del circuito; $c$ ) la fuerza electromotriz queda relegada exclusivamente para fenómenos de inducción electromagnética (ley de Faraday).

Una segunda confusión que también es frecuente en la enseñanza del electromagnetismo es una visión excesivamente dividida de los temas sin hacer mención a sus relaciones. Así la fuerza electromotriz se percibe más como un concepto unido a la inducción electromagnética y separado de fenómenos relacionados con circuitos de corriente continua y del concepto de diferencia de potencial. Se olvida que la fuerza electromotriz es una magnitud necesaria para una explicación lógico-racional del análisis energético de un circuito de corriente continua considerado como un sistema. Creemos que esta falta de relación entre conceptos de electromagnetismo ha quedado evidenciada en la cuestión 5 de nuestra investigación.

En resumen, mientras se olvide la introducción del concepto de fuerza electromotriz como una hipótesis de trabajo necesaria para una explicación coherente con un marco energetista de los circuitos de corriente continua y no se abra un debate sobre su significado en la enseñanza de la electricidad, será bastante difícil establecer estrategias de enseñanza que tengan como objetivo una mejor comprensión de las relaciones entre electrostática y electrocinética y, en concreto, de los circuitos eléctricos de corriente continua.

\section{NOTAS}

${ }^{1}$ En el caso que nos ocupa, podemos hablar de energía (potencial) química como de la energía interna del sistema, ya que la energía prácticamente se obtiene de la interacción de las sustancias químicas dentro de la pila y, en definitiva, por la variación en la estructura y configuración de los enlaces químicos de las sustancias iniciales. Sin embargo, es necesario indicar que el concepto de energía interna de un sistema es más complejo, ya que se define como la suma de las energías asociadas a las configuraciones cinética y potenciales posibles (estado físico, estructura química, estado eléctrico global, magnético, etc.).

${ }^{2}$ Sólo después de Maxwell, mediante los experimentos de Rowland, la identidad entre galvanismo y electricidad se considera experimentalmente demostrada. 


\section{REFERENCIAS BIBLIOGRÁFICAS}

AGUILAR, J. (2001). El legado de Galvani y Volta a la ciencia moderna. Revista Española de Física, 15(3), pp. 50-53.

ARCHIBALD, T. (1988). Tension and Potential from Ohm to Kirchhoff. Centaurus, 31, pp. 141-163.

ARONS, A.B. (1970). La evolución de los conceptos de la física. México: Trillas

BENSEGHIR, A. y CLOSSET, J.L. (1996). The electrostaticselectrokinetics transition: historical and educational difficulties. International Journal of Science Education, 18(2), pp. 179-191.

BROWN, T.M. (1969). The electrical current in Early Nineteenth-Century French Physics. Historical Studies in the Physical Sciences, 1, pp. 61-103.

BUCHWALD, J.Z. (1977). William Thomson and the Mathematization of Faraday's Electrostatics. Historical Studies in the Physical Sciences, 8, pp. 101-136.

BUCHWALD, J.Z. (1985). From Maxwell to microphysics, aspects of Electromagnetic. Theory in the Last Quarter of the Nineteenth Century. University of Chicago Press.

COHEN, K. et al. (1983). Potential difference and current in simple electric circuits: a study of student concepts. American Journal of Physics, 51(5), pp. 407-412.

CHABAY, R. y SHERWOOD, B. (2002). Matter and interactions (Vol. 2). Nueva York: John Wiley \& Sons, Icn.

COOK, T.D. y REICHARDT C.H.S. (1982). Qualitative and quantitative methods in evaluation research. Sage Publications, Inc.

DARRIGOL, O. (1998). Aux confins de l'ectrodynamique: Ions et électrons vers 1897. Revue d'Histoire des Sciences, 51(1), pp. 5-34.

DRIVER, R. et al. (1985). Children's Ideas in Science. Open University Press.

DUIT, R. y JUNG, W. (1985). Aspects of Understanding Electricity. Kiel: Christoph von Rhöneck.

DUIT, R. (1993). Research on Student's Conceptionsdevelopments and trends. «Third International Seminar on Misconceptions and Educational Strategies in Science and Mathematics». Cornell. Ithaca.

EYLON, B.S. y GANIEL, U. (1990). Macro-micro relationships: the missing link between electrostatics and electrodynamics in students' reasoning. International Journal of Science Education, 12(1), pp. 79-94.

FIGUIER, L. (1887). Les merveilles de l'électricité. Citado por Cardot. Association pour L'Histoire de l'électricité en France.

FOX, R. (1990). Laplacian Physics, Companion to the History of Modern Science. Routledge.

FRANKEL, E. (1977). J.B. Biot and the Mathematization of Experimental Physics in Napoleonic France. Historical Studies in the Physical Sciences, 8, pp. 33-72.
FURIÓ, C. y GUISASOLA, J. (1997). Deficiencias epistemológicas en la enseñanza habitual de los conceptos de campo y potencial eléctrico. Enseñanza de las Ciencias, 15(2), pp. 259-272.

FURIÓ, C. y GUISASOLA, J. (1998a). Construcción del concepto de potencial eléctrico mediante el aprendizaje por investigación. Revista de Enseñanza de la Física, 11(1), pp. 25-37.

FURIÓ, C. y GUISASOLA, J. (1998b). Difficulties on learning the concept of electric field. Science Education, 82(4), pp. 511-526.

FURIÓ, C. y GUISASOLA, J. (2001). La enseñanza del concepto potencial eléctrico basada en un modelo de aprendizaje como investigación orientada. Enseñanza de las Ciencias 19(2), pp. 319-334

FURIÓ, C., GUISASOLA, J., ALMUDÍ, J.M. y CEBERIO, M. (2003). Learning the electric field concept as oriented research activity. Science Education, 87, pp. 640-662.

GUERLAC, H. (1976). Chemistry as a Branch of Physics: Laplace's Collaboration with Lavoisier. Historical Studies in the Phisical Sciences, 7, pp. 193-276.

GIERE, R.N. (1991). Understanding scientific reasoning. Forth Worth, TX: Holt, Rinehart and Winston.

GIL, D. (1983). Tres paradigmas básicos en la enseñanza de las ciencias. Enseñanza de las Ciencias, 1(1), pp. 26-33.

GIL, D. et al. (1991). La enseñanza de las ciencias en la educación secundaria. Barcelona: ICE-Horsori.

GIL, D. (1991). ¿Qué hemos de saber y saber hacer los profesores de ciencias? Enseñanza de las Ciencias, 9(1), pp. 69-77.

GIL, D. (1993). Contribución de la historia y de la filosofía de la ciencia al desarrollo de un modelo de enseñanza-aprendizaje como investigación. Enseñanza de las Ciencias, 11(2), pp. 197-212.

HIERREZUELO, J. y MONTERO, A. (1989). La ciencia de los alumnos. Barcelona: Laia-MEC.

HUMPHREYS, A.W. (1937). The Development of the conception and measurement of electric current. Annals of Science, 2(2), pp. 164-178.

LAKATOS, I. (1970). Cristicism and the growth of knowledge. Londres: Cambridge University Press.

LANZARA, E. y ZANGARA, R. (1995). Potential difference measurements in the presence of a varying magnetic field. Physics Education, 30(2), pp. 85-89.

MARTÍN, J.(1999). «La introducción del concepto de campo en física». Tesis doctoral. Universidad de Valencia.

MARTÍN, J. y SOLBES, J. (2001). Diseño y evaluación de una propuesta para la enseñanza del concepto de campo en física. Enseñanza de las Ciencias, 19(3), pp. 393-404.

MONTERO, A. (2002). «Dificultades en el aprendizaje del 
concepto de fuerza electromotriz». Tesis de maestría. Universidad de Granada.

NERSESSIAN, N.J. (1995). Should physicist preach what they practice? Science Education, 4, pp. 203-226.

PAGE, C.H. (1977). Electromotive force, potential difference and voltage. American Journal of Physics, 45(10), pp. 978-980.

PANCALDI, G. (1990). Electricity and life. Volta's path to the battery. Historical Studies in the Physical and Biological Sciences, 21, pp. 123-160.

PEARCE, W. (1962). The physical sciences in the first half of the Nineteenth Century: Problems and sources. History of Science, 1, pp. 1-15.

PFUNDT, H. y DUIT, R. (1994). Bibliography: Student's alternative frameworks and science education. Institute for Science Education: University of Kiel.

PSILlOS, D. (1998). Connecting Research in Physics Education with Teacher Education. ICPE Book.

REITZ, J. et al. (1996). Fundamentos de la teoría electromagnética. Massachusetts: Addison-Wesley.

SATIEL, E. y VIENNOT, L. (1985). ¿Qué aprendemos de las semejanzas entre las ideas históricas y el razonamiento espontáneo de los estudiantes? Enseñanza de las Ciencias, 3(2), pp. 137-144.

SOLBES, J. y MARTÍN, J. (1991). Análisis de la introducción del concepto de campo. Revista Española de Física, 5(3), pp. $34-49$.

STEINBERG, M.S. (1992). What is electric potencial? Connecting Alessandro Volta and contemporary students.
Proceding of the Second International Conference on the History and Philosophy of Science and Science Teaching. Vol. II, pp. 473-480.

SUTTON, G. (1981). The politics of science in early Napoleonic france: The case of the voltaic pile. Historical Studies in the Physical Sciences, 11(2), pp. 329-366.

TATON, R. (1988). Historia general de las ciencias, 9. Barcelona: Orbis.

TOULMIN, R. (1972). Human understanding: the collective use and evolution of concepts. Pricenton University Press. Trad. cast. (1997). Madrid: Alizanza Editorial.

VARNEY, R.N. y FISHER, L.H. (1976). Contac potentials between metals: History, concepts and persistent misconceptions. American Journal of Physics, 44(5).

VARNEY, R.N. y FISHER, L.H. (1980). Electromotive force: Volta's forgotten concept. American Journal of Physics, 48(5), pp. 405-408.

VIENNOT, L. y RAINSON, S. (1999). Design and avaluation of a research-based teaching sequence: The superposition of electric field. International Journal of Science Education, 21(1), pp. 1-16.

WANDERSEE, J.H. (1992). The Historicality of cognition: Implications for Science Education Research. Journal of Research in Science Teaching, 29(4), pp. 423-434.

WISE, N.M. (1990). Electromagnetic Theory in the Ninetennth Century. Companion to the History of Modern Science. Routledge.

WHITTAKER, E. (1951). A History of the Theories of Aether and Electricity. Tomash Publishers.

[Artículo recibido en mayo de 2003 y aceptado en septiembre de 2004] 


\section{ANEXO}

1) Cuando se dice que «una carga eléctrica se desplaza entre los dos electrodos de una pila», varía:

a) su fuerza electromotriz;

b) su diferencia de potencial;

c) su potencial;

d) ninguna de las magnitudes anteriores.

Justifica tu elección.

2) Si la misma pila que se conecta a distintos circuitos, permanece constante:

a) la diferencia de potencial entre sus bornes;

b) la fuerza electromotriz de la pila;

c) la intensidad de la corriente que pasa por la pila;

d) ninguna de las anteriores.

Justifica la elección.

3) El concepto de potencial se introduce al estudiar las cargas en reposo (electrostática). Dicho concepto se aplica cuando las cargas están en movimiento (circuitos eléctricos) porque:

a) como se sabe, el movimiento de las cargas en los cables es insignificante debido a los choques de éstas con los restos positivos de la red metálica;

b) justifica la corriente que se establece entre dos puntos que, estando a diferente potencial, se conectan mediante un hilo conductor;

c) el concepto de diferencia de potencial entre dos puntos explica la aparición de la fuerza electromotriz;

d) no se trata del mismo concepto de diferencia de potencial en la electrostática que en el caso de circuitos eléctricos.

Justifica tu elección

4) Para el conjunto de un circuito cerrado, como el de la figura en el que se supone despreciable la resistencia interna de la pila, se cumple:

a) $\mathrm{V}=\mathrm{IR}$

b) $\xi=\mathrm{IR}$

c) las dos anteriores;

d) ninguna de las dos anteriores.

Justifica tu elección.

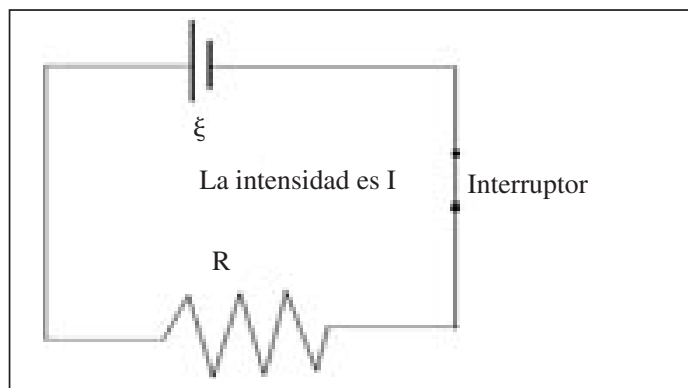

5) La figura representa un anillo de un material conductor en el seno de un campo magnético variable. En relación con esta situación, razona la veracidad o falsedad de las siguientes frases:

a) Hay fem que hace que la corriente se mueva por el anillo, pero no produce una d.d.p. entre dos puntos, como A y B de éste;

b) No hay d.d.p y, por tanto, no puede haber corriente en el anillo;

c) Si hay fem, debe haber una diferencia de potencial electrostático asociada a la misma que dará lugar a una corriente eléctrica;

d) Un voltímetro entre A y B no indicaría nada, un amperímetro intercalado en A indicaría paso de corriente.

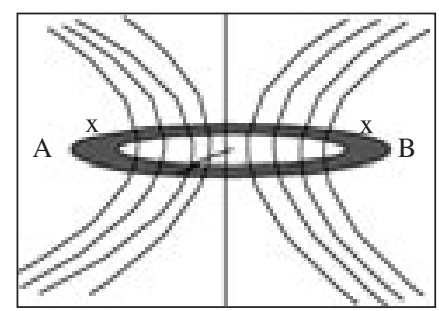

